\title{
PREFACE
}

\section{Humanitarian Audiology}

Humanitarian audiology may take many forms in many different places. It can be local in one's own community or in a different country. It may have many limitations and challenges; however, it can be extremely rewarding and have a lasting effect not only on those who receive assistance but also on those providing the service. I have been fortunate to be involved in an effort in Honduras over the last many years in an ongoing humanitarian effort and it has had a profound impact on me as a person as well as an audiologist.

My desire to participate in humanitarian efforts started many years ago when I spent time in a different capacity other than audiologist in Honduras. The people I met, the language that I learned, and the experiences that I obtained shaped me as a person and future audiologist. As I finished my degree and gained experience as an audiologist, I had a desire to return and use my new skills to help individuals with hearing loss in underserved countries. I sought out this opportunity, however, at the time I was not able to locate much direction on how to start on a project or what framework should be used to develop an effort. As these thoughts were marinating in my mind, an opportunity to return to Honduras as an audiologist presented itself and I jumped at the chance to return in a new capacity to a country that had been such an important part of my life.

Each trip, four in total up to this point, has brought new challenges and questions that are difficult to answer. Some questions include can this effort become sustainable, how to maintain funding, how to perform best practices when resources and equipment are limited, and how best to convey complex information in another language and to another culture. All of these questions do not have simple answers and will be different with each humanitarian effort whether that be in our own backyard or in another country.

With this experience in mind, I set out to discover how others involved in humanitarian audiology address these questions and the answers were very eye opening. This led to the articles of this edition. Some of the articles provide a framework to guide individuals on humanitarian projects, while others provide new and different solutions through advances in technology that were not available in the past. Other articles focus on different group's experiences with "boots on the ground" and how they have had to adapt to their unique circumstances. I am hopeful that this will provide guidance and new ideas to those who are involved in humanitarian audiology as well as those who are considering becoming involved.

I would not have been able to complete this edition of Seminars in Hearing without the help of many people. I would like to thank Catherine Palmer for taking a chance on me after I emailed her about the idea for this edition. I would also like to thank the authors for taking the time out of their busy lives to share information and experiences. Lastly, I would like to thank my wife Tara and children, Parker, Ivy, and Graham, for their support on this project.

Steven P. Smith, Au.D. ${ }^{1}$
${ }^{1}$ Division of Adult Audiology, Washington University School of Medicine, St. Louis, Missouri.

Address for correspondence: Steven P. Smith Au.D., Division of Adult Audiology, Washington University School of Medicine, Box 8115, 4566 Scott Avenue, St. Louis, MO 63110 (e-mail: stevensmith@wustl.edu).
Humanitarian Audiology; Guest Editor, Steven P. Smith, Au.D.

Semin Hear 2020;41:81-82. Copyright (C) 2020 by Thieme Medical Publishers, Inc., 333 Seventh Avenue, New York, NY 10001, USA. Tel: +1(212) 760-0888. DOI: https://doi.org/10.1055/s-0040-1708505. ISSN 0734-0451. 